\title{
Investigations on Shaped Mirror Systems in Quasi-Optical Mode Converters Based on Irradiance Moments Method
}

\author{
Hai Wang, ${ }^{1}$ Zejian Lu, ${ }^{1}$ Xiaoming Liu, ${ }^{1}$ Junsheng Yu, ${ }^{1}$ Yuan Yao, \\ Xiaodong Chen, ${ }^{2}$ Limei Qi, ${ }^{1}$ and Zhijiao Chen ${ }^{1}$ \\ ${ }^{1}$ BUPT-QMUL EM Theory \& Application International Research Lab, School of Electronic Engineering, \\ Beijing University of Posts and Telecommunications, Beijing 100876, China \\ ${ }^{2}$ School of Electronic Engineering and Computer Science, Queen Mary University of London, London E1 4NS, UK \\ Correspondence should be addressed to Hai Wang; wanghaibupt@163.com
}

Received 14 November 2015; Accepted 6 March 2016

Academic Editor: Safieddin Safavi-Naeini

Copyright (C) 2016 Hai Wang et al. This is an open access article distributed under the Creative Commons Attribution License, which permits unrestricted use, distribution, and reproduction in any medium, provided the original work is properly cited.

A method of transforming high-order Gaussian beams (GBs) mode into circular symmetry fundamental Gaussian beam (FGB) mode with arbitrary waist size is presented using irradiance moments method in quasi-optical (QO) mode converters. The double shaped mirrors correcting amplitude and phase simultaneously are generated by a single incidence irradiance sampling data and known ideal output FGB taking advantage of linear moment matching technique and Fresnel diffraction theory, which can be applied to a wide frequency range especially significant for terahertz band. The numerical coding procedure of creating double correcting mirrors and its fast convergence speed are discussed at $325 \mathrm{GHz}$. Numerical and experimental comparisons reveal the conclusion that enhancing surface precision and increasing moments order can improve main lobe levels.

\section{Introduction}

Gyrotrons are usually used in high power microwave (HPM) devices and excite higher-order cylindrical cavity mode $\mathrm{TE}_{m n}$ or $\mathrm{TM}_{m n}$. However, those modes cannot directly transmit in long distance due to nonlinear polarization and divergence of axial radiation [1-4]. What is worse, the existence of high-order spurious modes leads to the asymmetric, offsetfocus, and unstable amplitude and phase [5-9]. Quasi-optical (QO) mode converters with correction mirrors are widely utilized in such system to shape the electromagnetic field into desired fundamental Gaussian beam (FGB) $\mathrm{TEM}_{00}$ field distributions.

Traditionally, for effectively suppressing beam divergence and focusing adjustment, the QO mode converters usually adopt regular mirrors with quadratic surfaces to transform high-order modes into low-order modes, such as parabolic and elliptic mirrors. However, in some applications, the quality of the output beam from regular mirrors cannot meet the requirements of pure FGB distributions. For example, although the power-conversion efficiency of $220 \mathrm{GHz}$ Denisov-type QO mode convertor is about $95 \%$ in [9], the output beam still can be corrected. The conversion efficiency of Vlasov-type QO mode convertor for a $0.42 \mathrm{THz}$ in [10] is $88 \%$. So the conversion efficiency needs to be further improved, and the output beam should be effectively converted into the desired FGB profile by introducing shaped correcting mirrors system, which are often irregular structures created by iterative algorithms. There are many theoretical and experimental investigations on shaped correcting mirrors system [11, 12], such as Katsenelenbaum-Semenov algorithm (KSA) method [13-18], the phase gradient method [19], wave-beam shaping technique [20], and the irradiance moments method [21-24]. The goal of KSA method is to obtain the minimum deviation of beam energies between the known incident field and desired output field passing through each mirror. This algorithm needs to be calculated separately for each mirror, and the iterative result of one mirror directly affects the generation of next mirror. Moreover, the timeconsuming and optimization results largely depend on the choices of initial perturbed surface.

When it knows the incident electric field and the reflected electric field of each mirror, the phase gradient method 
based on the Fast Fourier Transform (FFT) can be applied to obtaining the express of correction mirror. The perfect electric conductor (PEC) mirror phase corrector has been designed in the sub-THz gyrotron in [19], where it is suitable for smooth phase front for solving derivative directly on the phase difference. The wave-beam shaping technique needs to calculate the forward-backward-forward field by multiple circulatory iterations taking advantage of plane wave expansion for calculation with FFT. The iterative is completed when the wave-beam profiles converge and its typical iteration number is 50-100 times [20].

Irradiance moments method, which is originally utilized for phase retrieval based on amplitude measurement data at several planes $[21,22]$, has also been successfully demonstrated in mirror synthesis in a $110 \mathrm{GHz}$ gyrotron internal mode converter [24]. The correcting mirrors synthesized using the irradiance moments are easy to fabricate because the surface profiles are smooth when compared with surfaces created by the KSA algorithm in [18], making a huge advantage in comparison to the mirrors synthesized using other iteration methods, where the mirror surfaces have to be smoothed out using the phase unwrapping and filtering procedures [25]. However, There are still some problems that remain to be unsolved when applying the irradiance moments method into terahertz $(\mathrm{THz})$ wave band. The increasing frequency posts strict demands on manufacture of the system, which could lead to more stray high-order modes. In this paper, several important parameters are investigated, such as (a) the number of moments orders adapted in the iterative process, (b) the proportion of high-order GBs mode, (c) the mirror manufacturing error, and (d) the available working bandwidth especially in high frequency.

In this paper, the characteristics of irradiance moment method are overall investigated, and the numerical example of a double shaped mirrors system analyzing different mode ratio of the first fourth high-order GBs mode is discussed in $\mathrm{THz}$ band. The resolution ability of different moments order is also examined according to the quality of reflection field involving double conversion coefficients and main lobe levels. The comparison results among window fields obtain the effect of surface roughness and suitable bandwidth.

This paper is organized as follows. The principle of irradiance moments method and coding procedure of numerical optimization creating double shaped mirrors are viewed in Section 2. The double correcting mirrors system and related parameters analysis on the irradiance moments method are detailedly described in Section 3. Finally, we discuss and summarize all of the work.

\section{Theory and Implementation of Irradiance Moments Method}

The location relationship between double correcting mirrors system utilized in QO mode convertor and the conversion process of FGB is depicted in Figure 1, where Mirror 1 and Mirror 2 are shaped mirrors to be determined. In this system, the field on the incident plane radiates out into Mirror 1 with an angle $\theta$ and then is reflected from Mirror 2 into the

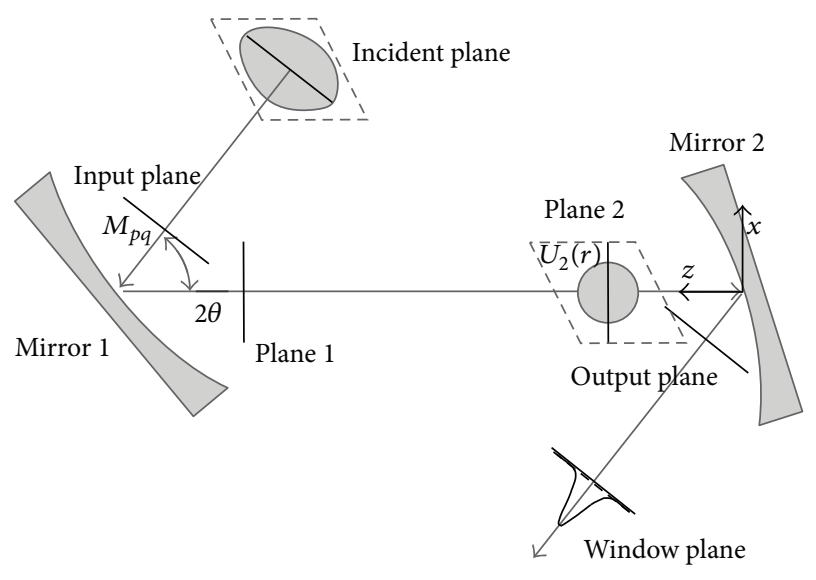

FIGURE 1: The location relationship between double shaped correcting mirrors and the conversion process of the FGB.

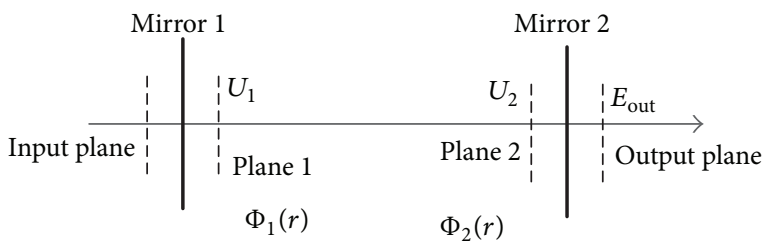

FIGURE 2: The schematic diagram of equivalent wave path model of double correcting mirrors system.

window plane. Generally, the field on the incident plane can be known by either direct measurement of the amplitude and phase distributions on the plane or, for those cases where only amplitude distribution can be measured, some phase retrieval methods being available to restore the phase information on the plane via sampling two or more arbitrary measurement planes $[26,27]$. The field on the window plane is the desired FGB that should have characteristics of the circular symmetry amplitude, low side lobe levels, and stable phase center.

The correcting mirror system can be regarded as an optimization module in amplitude and phase. According to the difference of input and output field, it needs to design light path by mirrors for obtaining desired FGB. Therefore, the problem stated in here can be simplified equivalently to the wave path model as shown in Figure 2, where $\left|U_{1}\right|$ and $\left|U_{2}\right|$ are the amplitude distributions while $\Phi_{1}(r)=\arg \left(U_{1}\right)$ and $\Phi_{2}(r)=\arg \left(U_{2}\right)$ are the phase distributions on plane 1 and plane 2 , respectively.

In the equivalent model, the electromagnetic field propagates from Mirror 1 to Mirror 2. In order to synthesize Mirror 1 and Mirror 2, the phase distributions on plane 1 and plane 2 have to be determined. The fields on the input plane and plane 1 are corresponding to incident and reflected fields from Mirror 1 while the fields on plane 2 and output plane are relative to Mirror 2. The field on the input plane can be obtained by propagating the original field on the incident plane forward. Meanwhile the field on the output plane is calculated via propagating the field on the window plane backward. The amplitude distributions on plane 1 and plane 2 are identical to amplitude distributions on the input plane and 
output plane. The location points of discrete mirrors surfaces are derived, respectively, according to the phase relationship of incident and reflected field in their own mirror coordinate systems.

The irradiance moment $M_{p q}\left(z_{0}\right)$ over the input plane and its propagation formula $M_{p q}(z)$ are characterized with twodimensional moments $[22,24]$ :

$$
\begin{aligned}
& M_{p q}\left(z_{0}\right)=\frac{M_{p q}\left(x^{p}, y^{q}, z_{0}\right)}{M_{00}\left(z_{0}\right)} \\
& \quad=\frac{\iint_{-\infty}^{+\infty} x^{p} y^{q}\left|U_{z}(r)\right|^{2} d x d y}{\iint_{-\infty}^{+\infty}\left|U_{z}(r)\right|^{2} d x d y}, \\
& M_{p q}(z, \phi) \\
& \quad=\left(-i \frac{z}{k}\right)^{p+q} \iint_{-\infty}^{+\infty} U G \frac{\partial^{p+q}}{\partial x^{p} \partial y^{q}}(U G)^{*} d x d y,
\end{aligned}
$$

where the amplitude of $\left|U_{z}\right|^{2}$ is the irradiance intensity at input plane and $G(r, z)$ is the Green function in free space. The subscripts of $p$ and $q$ represent different moments order in the $x$ and $y$ direction. Thus the total moments order number $N$ is $p+q$. The denominator $M_{00}\left(z_{0}\right)$ corresponds to the zero-order moment representing total beam energy, and $M_{p q}\left(z_{0}\right)$ is also called the average value of irradiance energy. For a collimated light beam, the wave equation with Gaussian shape at plane 2 can be expressed as

$$
\begin{aligned}
& U_{2}(r)=P_{2}(r) \exp \left[i \varphi_{2}(r)\right], \\
& \varphi_{2}(r)=\sum_{j=1}^{N_{\max }} \sum_{k=0}^{j} \frac{\phi_{j-k, k} x^{j-k} y^{k}}{L^{j+k}},
\end{aligned}
$$

where $P_{2}(r)$ is the Gaussian amplitude distribution and $2 L$ is the dimension of the plane. The phase distribution $\varphi_{2}(r)$ is an oblique upper triangular matrix except for zero order and should be iterated out by moment propagation theorem [22]. The set $\left\{\phi_{m n}\right\}$ is polynomial coefficient with dimensionless constants and can be solved out by the moments $M_{p q}$. The number of $N_{\max }$ depends on the same total moments order number. The total number of the phase terms is $N_{t}=$ $N_{\text {max }}\left(N_{\max }+3\right) / 2$. When $N_{\max }=3, N_{\max }=4$, and $N_{\max }=5$, the phase terms, which are to be discussed in detail, involve $N_{t}=9, N_{t}=14$, and $N_{t}=20$ polynomial coefficients, respectively.

Using (2), the moments in arbitrary location from plane 2 to plane 1 can be represented. According to the known incidence moment information in (1), the next step is to find a convergence way that makes the differences between $M_{p q}(z)$ and $M_{p q}(z, \phi)$ smallest, which is related to variables set $\left\{\phi_{m n}\right\}$.

When it begins the first iteration, the phase in plane 2 can take a guess that the phase polynomial coefficients $\left\{\phi_{m n}\right\}$ are given the same number as initial iteration condition. By evaluating the derivative of function $M_{p q}(z, \phi)$ with respect to every set element $\left\{\phi_{m n}\right\}$ and calculating the differences $\Delta M_{p q}$ between the $M_{p q}(z, \phi)$ and $M_{p q}\left(z_{0}\right)$, the changes of set $\left\{\Delta \phi_{m n}\right\}$ can be found by solving the matrix equations. What is more, the new set $\left\{\phi_{m n}+\Delta \phi_{m n}\right\}$ can be made up in plane 2 for continuing to start next iteration until set convergence. Due to the fact that the set $\left\{\phi_{m n}\right\}$ is real value, the real number of iteration matrix equation can be expressed as follows[24]:

$$
\begin{aligned}
\operatorname{Re}( & {\left.[B]_{N_{t} \times N_{t}}\right) \cdot\left[\Delta \phi_{m n}\right]_{N_{t} \times 1}=\operatorname{Re}\left(\left[\Delta M_{p q}\right]_{N_{t} \times 1}\right), } \\
B= & \frac{\partial M_{p q}(z, \phi)}{\partial \phi_{m n}}=\left(-i \frac{z}{k}\right)^{p+q} \iint_{-\infty}^{+\infty} d x d y \\
& \cdot\left\{i \frac{x^{m} y^{n}}{L^{m+n}} U G \frac{\partial^{p+q}(U G)^{*}}{\partial x^{p} \partial y^{q}}\right. \\
& \left.+U G \frac{\partial^{p+q}\left[-i\left(x^{m} y^{n} / L^{m+n}\right)(U G)^{*}\right]}{\partial x^{p} \partial y^{q}}\right\} .
\end{aligned}
$$

The linear matrix can be solved via Newton iteration method and the iterative convergence criteria are that the square sum of amplitude $\Delta M_{p q}$ is close to zero. Once the polynomial coefficient set $\left\{\phi_{m n}\right\}$ is solved, the field on plane 2 can be determined and the field in plane 1 can be obtained by the Rayleigh-Sommerfeld diffraction integral formula in [28]

$$
\begin{gathered}
U_{1}\left(x_{1}, y_{1}, z_{1}\right)=\frac{1}{2 \pi} \iint_{-\infty}^{+\infty} U_{2}\left(x_{2}, y_{2}, z_{2}\right) \\
\frac{\exp (j k r)}{R} \frac{z_{1}-z_{2}}{R}\left(\frac{1}{R}-j k\right) d x_{2} d y_{2}, \\
E_{\text {window }}(x, y)=\sqrt{\frac{2}{\pi w_{0}^{2}}} \exp \left(-\frac{x^{2}+y^{2}}{w_{0}^{2}}\right),
\end{gathered}
$$

where $R$ is the integral points distance between double planes. According to (5) and input plane filed, the phase distribution variation passing the first mirror can be obtained. In the same principle, we can obtain the back propagation field $E_{\text {out }}$ reflected from the second mirror by the RayleighSommerfeld diffraction integral only using the window plane field (6), and the phase variation passing the second mirror can also be obtained. Here, the window field is a FGB, the parameters of which, such as the position of window and the size of beam waist $w_{0}$, are chosen according to practical applications.

When the total four plane fields have been solved, the coordinate position of double correcting mirrors profiles $\left(x^{\prime}, y^{\prime}, z^{\prime}\right)$ with a certain incidence angle $\theta$ can be created by the difference of phase. However, the field calculated directly is wrapped phase, where the range becomes $(-\pi,+\pi)$ and leads to discontinuous surfaces, so it needs to manually add the appropriate $2 \pi \cdot g(r)$ to become the unwrapped phase with new range $(-\infty,+\infty)$, where $g(r)$ has to be integer values. An example of phase process of $\exp \left(j 5 x^{2}\right)$ transformation wrapped phase into unwrapped phase can be found in [25]. The double correcting unwrapped phase now can be generated according to (7) and (8). The threedimensional correcting mirrors profiles decided by phase shift can be obtained by (9), where the $y$-axis is system symmetry axis. The unwrapped process of wrapped phase is coded in Algorithm 1. The corresponding numerical coding 
procedure of irradiance moments method is shown as in Figure 3:

$$
\begin{aligned}
\Delta \Phi_{\mathrm{I}}(r) & =\left[\Phi_{1}(r)-\Phi_{\text {in }}(r)\right]+2 \pi \cdot g_{\mathrm{I}}(r), \\
\Delta \Phi_{\mathrm{II}}(r) & =\left[\Phi_{\text {out }}(r)-\Phi_{2}(r)\right]+2 \pi \cdot g_{\mathrm{II}}(r), \\
{\left[\begin{array}{c}
x^{\prime} \\
y^{\prime} \\
z^{\prime}
\end{array}\right] } & =\left[\begin{array}{ccc}
\frac{1}{\cos \theta} & 0 & 0 \\
0 & 1 & 0 \\
0 & 0 & \frac{1}{2 k \cos \theta}
\end{array}\right]\left[\begin{array}{c}
x \\
y \\
\Delta \Phi
\end{array}\right] .
\end{aligned}
$$

$$
\begin{aligned}
\eta_{s} & =\frac{\iint_{S}\left|f_{1}\right| \cdot\left|f_{2}\right| d r}{\sqrt{\iint_{S}\left|f_{1}\right|^{2} d r \cdot \iint_{S}\left|f_{2}\right|^{2} d r}}, \\
\eta_{v} & =\frac{\iint_{S}\left|f_{1}\right|\left|f_{2}\right| \exp \left[i\left(\phi_{1}-\phi_{2}\right)\right] d r \cdot \iint_{S}\left|f_{1}\right|\left|f_{2}\right| \exp \left[i\left(\phi_{2}-\phi_{1}\right)\right] d r}{\iint_{S}\left|f_{1}\right|^{2} d r \cdot \iint_{S}\left|f_{2}\right|^{2} d r} .
\end{aligned}
$$

For comparing the consistency between the real reflected fields $f_{1} \cdot \exp \left(j \phi_{1}\right)$ after every mirror and the theoretical design values $f_{2} \cdot \exp \left(j \phi_{2}\right)$, both the parameters that scalar and vector conversion efficiency formula use (10) on behalf of beam coupling in energy and direction should be selected. They should be referenced at the same time because their center phase of main lobe may be likely to have significant deterioration although it sometimes has very high value of recovered energy:

\section{Numerical Results and Comparison}

A double correcting mirrors system working at the center frequency of $325 \mathrm{GHz}$ is synthesized in this chapter based on the irradiance moments method. The double correcting mirrors have the function of adjusting amplitude and phase simultaneously. The presented irradiance moments method is implemented in a MATLAB code and the system structure is created in commercial simulation software GRASP.

3.1. The Geometry Model of the Correcting Mirrors System. The two and three dimensions' geometry model of the double correcting mirrors system is depicted in Figure 4. The distance between feed and the first mirror is $D 1=$ $6.23 \mathrm{~cm}$, and the double mirrors have the distance of D2 = $8.31 \mathrm{~cm}$. The surfaces are defined by local coordinate systems $\left(x_{1}, y_{1}, z_{1}\right)$ and $\left(x_{2}, y_{2}, z_{2}\right)$ in Figure 4 at the center reflection points specified by $D 1$ and $D 2$ in system, where sign(+) and $\operatorname{sign}(-)$ express rotation direction of $z$-axis. The dimensions of both mirrors are $6.71 \mathrm{~cm} \times 5.81 \mathrm{~cm}$ in rectangle shape and there are 14641 discrete coordinate points in total for each mirror, where sampling distance between half and one wavelength is advisable. It is worth remembering that the truncated dimension of the mirror should be larger than beam waist for avoiding edge diffraction as far as possible. The window plane is chosen at a distance of $D 3=9.84 \mathrm{~cm}$ away from Mirror 2. The desired beam waist is $w_{0}=6 \lambda$, which can be adjusted according to the GB propagation theory.

The correcting shaped mirrors are synthesized via the procedure presented in Figure 3, while the whole system is validated using Physical Optics (PO) method in GRASP. In practice, the radiated field from the radiator can be decomposed into a superposition of various GB modes. Therefore, in order to simulate the actual output field of radiators, a mixture of the fundamental and high-order GBs mode by applying different waist radius is utilized as the feed.
The selection of GBs mode combination with different waist radius greatly affects the distortion of the feed from FGB. The zero-order and high-order GBs mode in the rectangular coordinate [29] are shown as

$$
\begin{gathered}
E_{m n}(r, z)=\left(\frac{1}{\pi \omega_{x} \omega_{y} 2^{m+n-1} m ! n !}\right)^{0.5} H_{m}\left(\frac{\sqrt{2} x}{\omega_{x}}\right) \\
\cdot H_{n}\left(\frac{\sqrt{2} y}{\omega_{y}}\right) \cdot \exp \left(-\frac{x^{2}}{\omega_{x}^{2}}-\frac{y^{2}}{\omega_{y}^{2}}\right) \cdot \exp [-j(k z \\
\left.\left.+\frac{\pi x^{2}}{\lambda R_{x}}+\frac{\pi y^{2}}{\lambda R_{y}}-\frac{(2 m+1) \phi_{0 x}}{2}-\frac{(2 n+1) \phi_{0 y}}{2}\right)\right],
\end{gathered}
$$

where $\omega_{x}$ and $\omega_{y}$ denote the beam radius, $R_{x}$ and $R_{y}$ describe the radius of curvature, and $\phi_{0 x}$ and $\phi_{0 y}$ express the phase shift in the $x$ and $y$ direction, respectively. The function of $H_{m}(x)$ and $H_{n}(y)$ represents the Hermite polynomials. The field on the incident plane composes zero-order mode $\mathrm{GB}(0,0)$ and high-order modes $\mathrm{GB}(m, n)$ with different waist radius on the $x$ and $y$ direction and then is inputted into the GRASP software as the feed in tabulated form.

Considering different component content of elliptic high-order GBs mode [see (11)], we select $\mathrm{GB}(0,0)$ and the first fourth high-order GBs mode under the ratio of $0.7: 0.06: 0.03: 0.02: 0.002$ as inputted feed field, and the total number of GBs mode is $15(1: 2: 3: 4: 5)$. The inputted zeroorder mode $\mathrm{GB}(0,0)$ of feed field has $\omega_{x}=8 \lambda$ and $\omega_{y}=$ $10 \lambda$ in the $x$ and $y$ direction, respectively, but the waists of high-order modes vary from $6 \lambda$ to $15 \lambda$ for presenting the asymmetry of the feed field. The desired beam waist on the window is $\omega_{0}=6 \lambda$ at the center frequency of $325 \mathrm{GHz}$. The scalar and vector conversion efficiency between the inputted feed field and the desired window field are only $88.84 \%$ and $78.79 \%$, respectively.

The presented irradiance moments algorithm converges after seven iterations. Using (7), (8), and (9), the double 


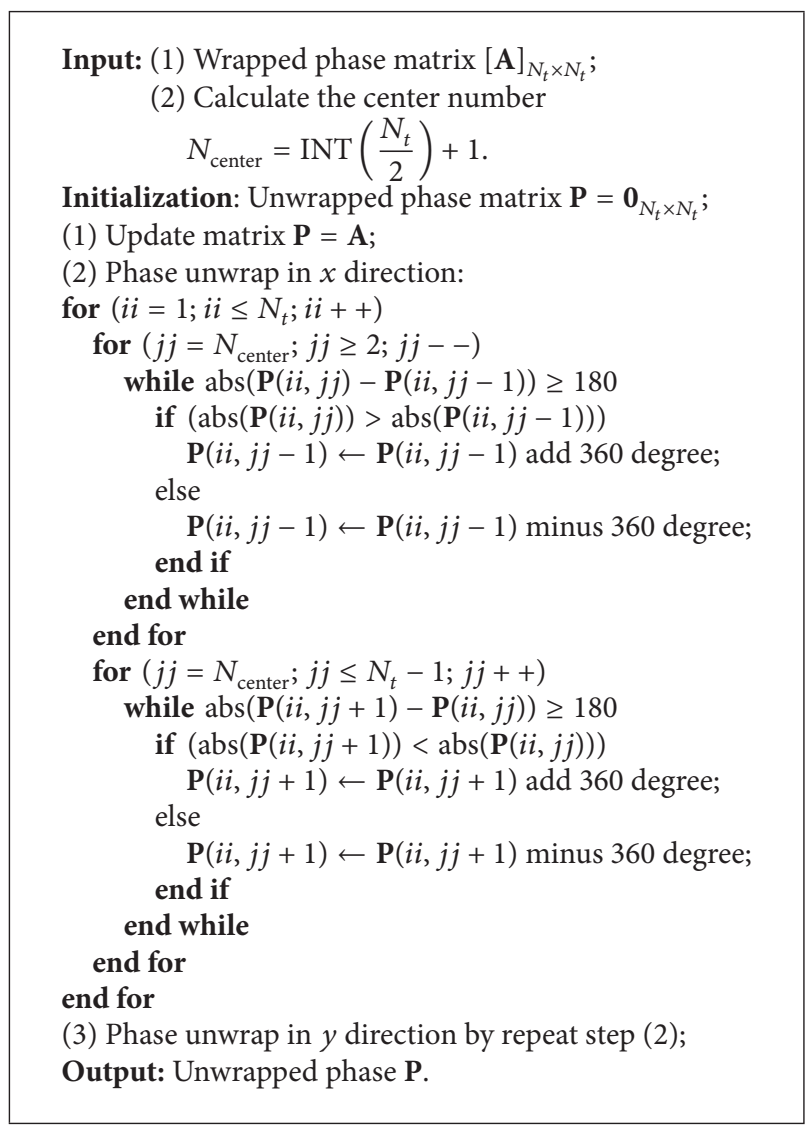

ALGORITHM 1: The unwrapped process of wrapped phase precoding.

shaped correcting mirrors created in $\theta=45$ degrees by irradiance moments method are shown in Figure 5. The feed field is transformed into a field specified in a plane to equivalent currents in the same plane as tabulated planar source in GRASP. These currents are integrated when the radiated field is to be determined. The system surfaces are defined through interpolation between tabulated $z$-values given at irregularly spaced $(x, y)$-points from discrete coordinate points. The actual beam transmission and transformation passing through double correcting mirrors have been shown in Figure 6, where the left is normalized amplitude $(\mathrm{dB})$ and the right is its phase comparing theoretical field with simulation values. In Figures 6(a)-6(b), the feed field is made up of $15 \mathrm{GBs}$ mode with tilted amplitude and asymmetrical phase. Figures 6(c)-6(d) are reflection field passing through distance of $D 2$, where the beam waist is $10 \lambda$ and it has linear phase in accordance with theoretical change tendency. The window field is obtained through $D 3$ in Figures 6(e)-6(f), where the beam waist is $6 \lambda$, and the phase in main lobe range $(1 \mathrm{~cm} \times 1 \mathrm{~cm})$ is circular symmetrical like the change of GB amplitude distribution.

In the process of validation in GRASP, the discrete coordinate points are inputted into the software to construct the mirror surfaces. However, if the double mirrors are mounted according to the original coordinate system in Figure 4 , the output field on the window is found to be

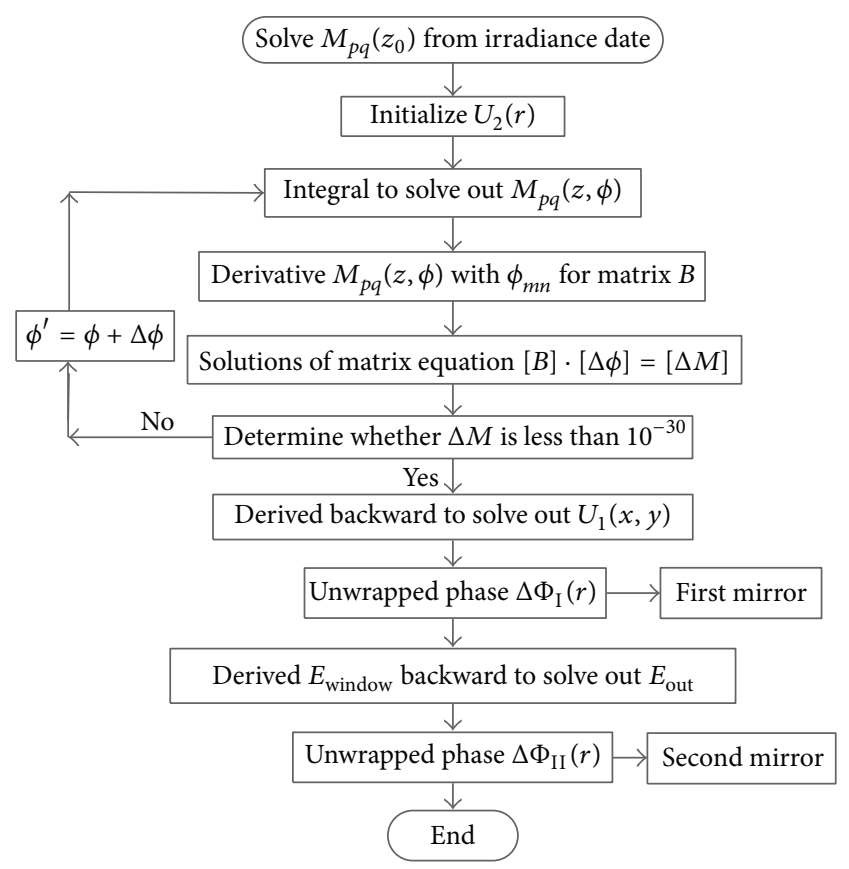

FIGURE 3: Numerical coding procedure of irradiance moments method creating double correcting mirrors.

slightly off-center in the simulation, which leads to a loss of conversion efficiency. This phenomenon is caused by discretization mirror surfaces, where they do not completely satisfy the law of reflection for irregular shape. However, the problem can be solved by slightly adjusting the orientation of double mirrors in the opposite direction. According to Figure 4(a), selecting mirror coordinate axes $z_{1}$ and $z_{2}$ as the rotation axis is to change their directions meanwhile guaranteeing that the $y$ direction is constant. Obviously, when the zero-order mode $\mathrm{GB}(0,0)$ becomes lower, the perturbed surface causes the greater rotation angle. For example, when the ratio of $\mathrm{GB}(0,0)$ is 0.7 , it needs to adjust the angle $\left(\Delta \theta z_{1}, \Delta \theta z_{2}\right)=\left(+0.2997^{\circ},-0.293^{\circ}\right)$, respectively, but selects the angle $\left(\Delta \theta z_{1}, \Delta \theta z_{2}\right)=\left(+0.6426^{\circ},-0.5807^{\circ}\right)$ at the case of 0.56 .

3.2. The Influence of Incident Angles on the Window Field. In (9), the final discrete data points of mirrors surface depend on the incident angle $\theta$. Considering the blockage effect between double mirrors and in order to fully receive the field energy, the incident angle $\theta$ can be selected reasonably in the range of 30 50 degrees. Comparison simulation results of window field between scalar and vector conversion efficiency with different input angles and the position of the first side lobe about normalized copolarization amplitude are shown in Table 1. There are minor differences between such results and they could be caused by simulation error, so the incident angles cannot largely change the performance of the window field. In this case, unless the incident angles are otherwise expressed, all the simulation results are obtained in the case of 45 degrees. 


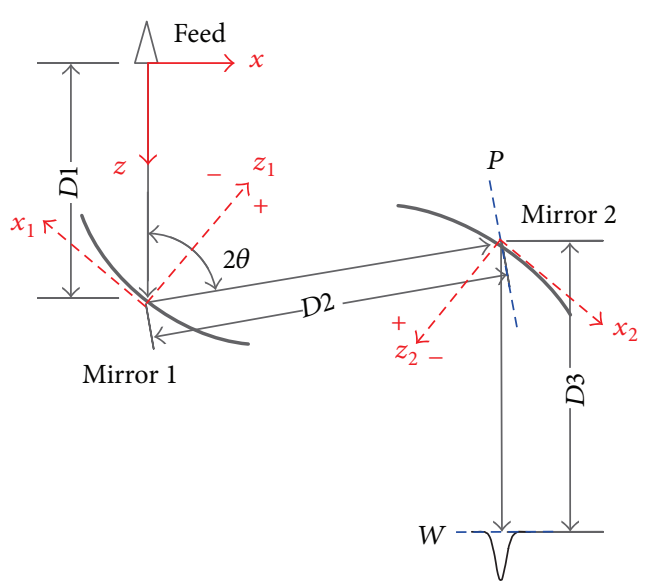

(a)

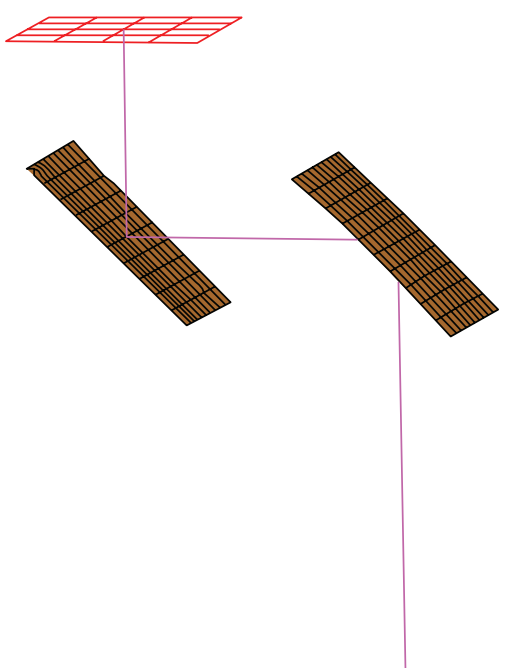

(b)

FIGURE 4: The geometry model of the correcting mirrors system: (a) coordinate system and structural parameters; (b) three-dimensional simulation model $(\theta=45 \mathrm{deg})$ in GRASP.

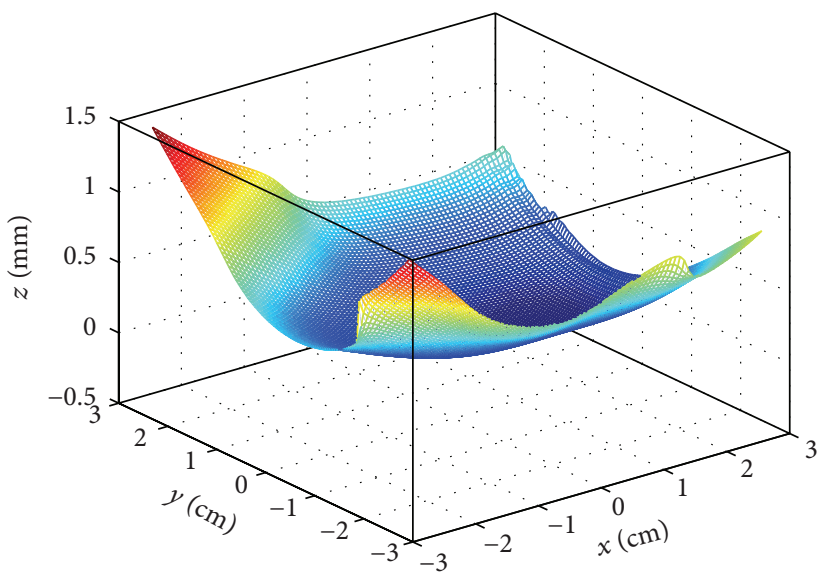

(a)

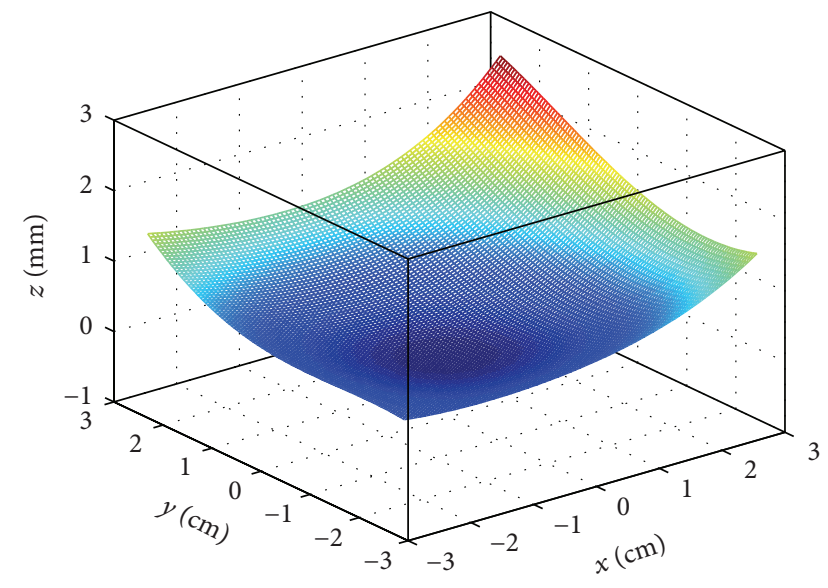

(b)

FIGURE 5: The double shaped correcting mirrors are created by irradiance moments method at $325 \mathrm{GHz}$ with $\theta=45$ deg and GBs ratio $0.7: 0.06: 0.03: 0.02: 0.002$. (a) is the first correcting mirror. (b) is the second correcting mirror.

TABLE 1: The influence of incident angles on the output window field.

\begin{tabular}{lccc}
\hline Input angle & $\eta_{s} D 3$ & $\eta_{v} D 3$ & Side lobe levels \\
\hline $30 \mathrm{deg}$ & 0.989361 & 0.941582 & $-42.12 \mathrm{~dB}$ \\
$35 \mathrm{deg}$ & 0.990832 & 0.945805 & $-42.31 \mathrm{~dB}$ \\
$45 \mathrm{deg}$ & 0.990953 & 0.948277 & $-42.82 \mathrm{~dB}$ \\
$50 \mathrm{deg}$ & 0.987739 & 0.938693 & $-41.03 \mathrm{~dB}$ \\
\hline
\end{tabular}

3.3. The Convergence Speed of the Irradiance Moments Method. The convergence speed of the irradiance moments depends on the proportion of different GBs mode and the order of moments chosen. The relationship among the iteration times, the convergence speed, and the computation time with the different component of GBs mode and the order of moments is shown in Figure 7. In the picture, the horizontal axis represents the iteration times and the left vertical axis is the convergence criteria while the right vertical axis corresponds to the computation time in hours. In both pictures, the solid lines are mapped based on the number of high-order GBs mode $(m+n$ is less than $2,3,4$, and 5 where it is marked as $2 \mathrm{M}, 3 \mathrm{M}, 4 \mathrm{M}$, and $5 \mathrm{M}$ ), the ratio of elliptic zero-order mode $\mathrm{GB}(0,0)$ in the mixed fields (marked as 0.40 , 0.56 , and 0.70 ), and the moments order (marked as 3rd, 4th, and 5th); meanwhile the dotted line having same color and marker symbol is its corresponding iteration time (hours).

As it is shown in the picture, the iteration times increase when the order of moments is improving. The algorithm can always converge for the first fourth-order GBs mode within 6 14 iteration times. With the same moments order, the lower the mode component of $\operatorname{GB}(0,0)$ is, the slower the convergence speed it has. However, when the component of 


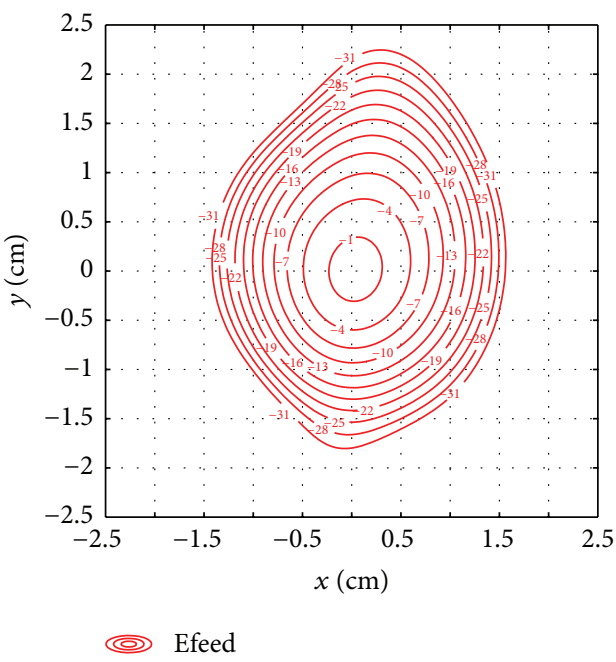

(a) Amplitude

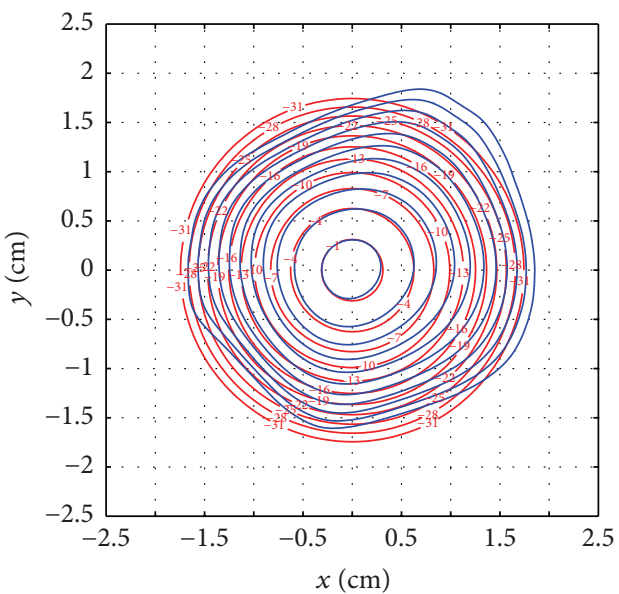

(a) Eplane 2

Es mirror 2_co

(c) Amplitude

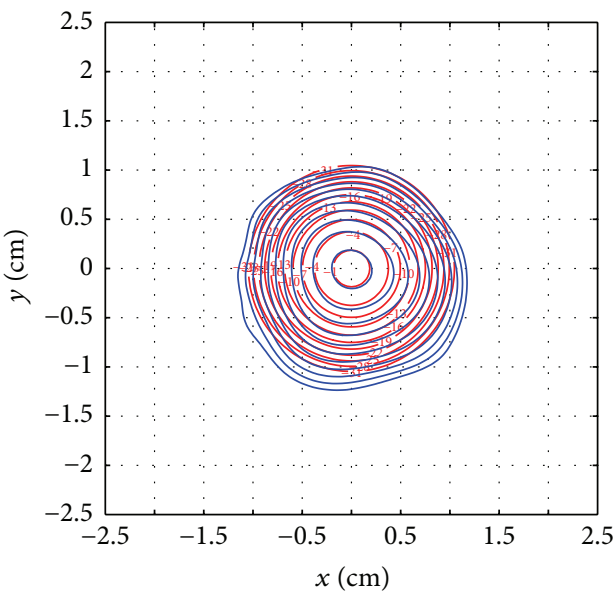

(a) Ideal FGB

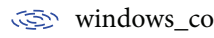

(e) Amplitude

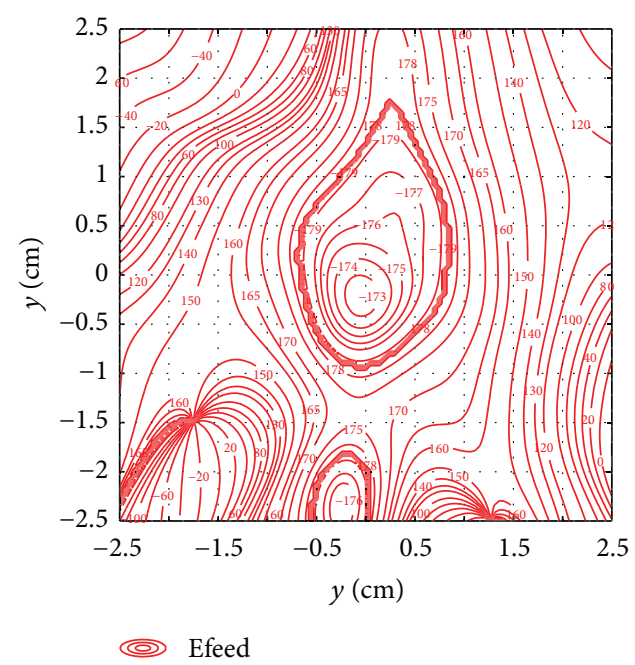

(b) Phase

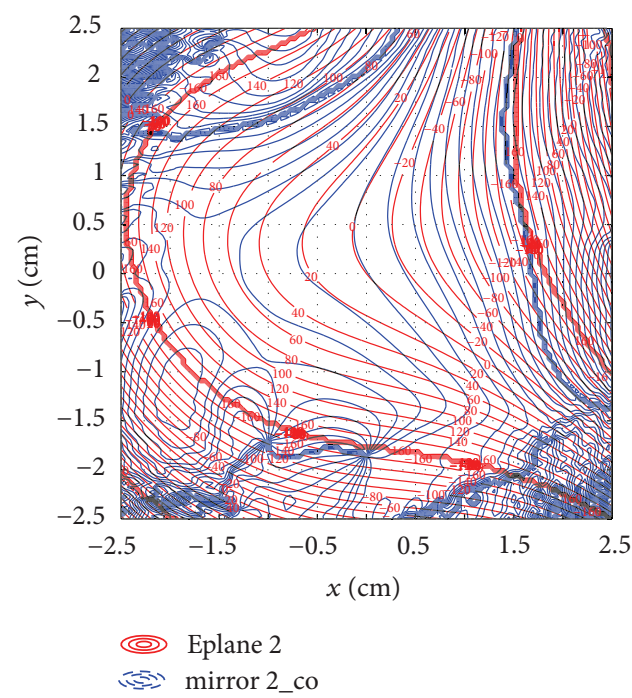

(d) Phase

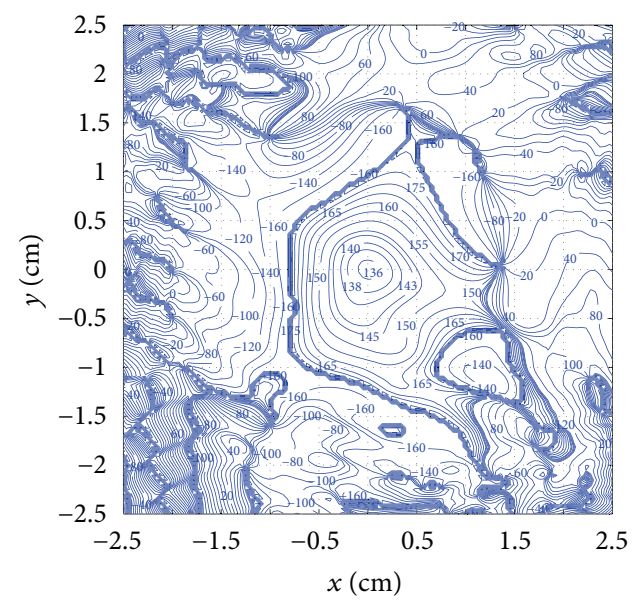

(1) windows_co

(f) Phase

FIGURE 6: The actual beam transmission and transformation process passing through double correcting mirrors. The left is normalized amplitude $(\mathrm{dB})$ and the right is phase with theoretical (red line) and simulation (blue line) values. (a)-(b) are the feed field, (c)-(d) are the reflected field from the first mirror after $D 2$, and (e)-(f) are the window field $\left(\omega_{0}=6 \lambda\right)$ reflected from the second mirror after $D 3$. 


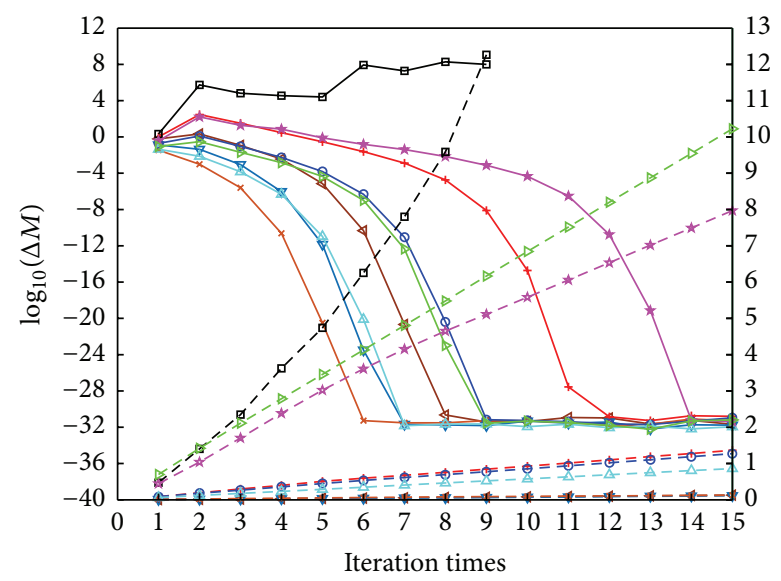

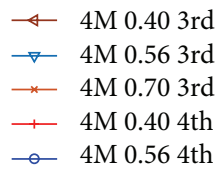

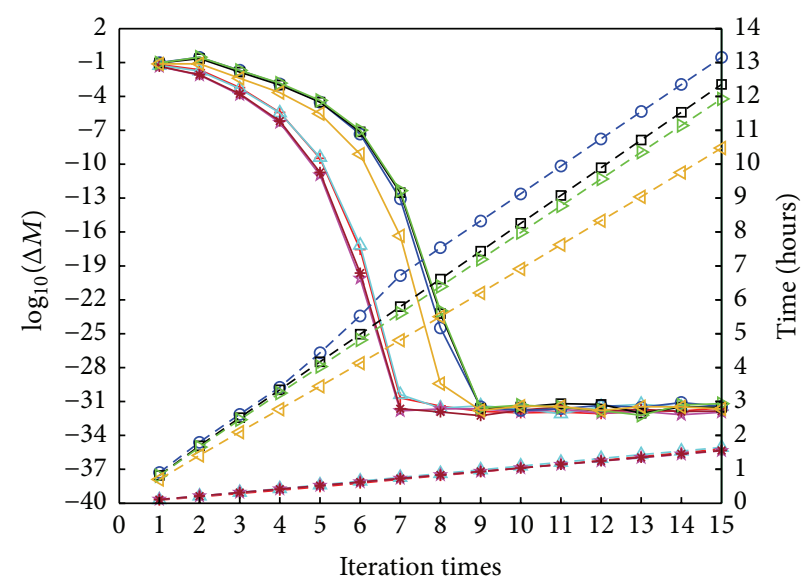

$\begin{array}{ll}\rightarrow \text { 2M 0.7 4th } & - \text { 2M 0.7 5th } \\ - \text { 3M 0.7 4th } & -3 \mathrm{M} \mathrm{0.75th} \\ \star \text { * 4M 0.7 4th } & \rightarrow \text { 4M 0.7 5th } \\ \rightarrow-5 \mathrm{M} \mathrm{0.7} \mathrm{4th} & -5 \mathrm{M} \mathrm{0.75th}\end{array}$

(b)

FIGURE 7: The relationship among the iteration times, the convergence speed, and the computation time. The horizontal axis is iteration times. In vertical axis, the left is convergence speed $\left(\log _{10}(\Delta M)\right)$, and the right is iteration time (hours): (a) keeps same mode number but has different ratio of $\mathrm{GB}(0,0)$ and moments order number; (b) keeps same ratio of $\mathrm{GB}(0,0)$ with 0.7 but increases the number of high-order $\mathrm{GBs}$ mode and moments order number.

$\mathrm{GB}(0,0)$ mode is only 40 percent, the fifth order of moment does not converge because it becomes difficult to restore the accurate phase distribution of fundamental mode when its incident content is very low. From the black line with square maker in Figure $7(\mathrm{a})$, the value of $\Delta M$ rises from the first time and then it begins to float in $10^{4} \sim 10^{8}$ all the time during 12 hours with 9 times. This also shows that the increasing number of moments order does no good to the lower $\mathrm{GB}(0,0)$ situation. If the input field ensures the same ratio of $\operatorname{GB}(0,0)$ with 0.7 and meanwhile increases the number of high-order modes, the convergence speed becomes faster. Obviously, the third-order moment has the fastest convergence speed, and the convergence speed of the fifth-order moment is 7 9 times that of the fourth order.

The required computational time is calculated with $121 \times$ 121 complex data using CPU i7-2600@3.4 GHz and RAM $12 \mathrm{~GB}$ in 64-bit operating system.

3.4. The Convergence Speed of the Irradiance Moments Method. The comparison results of window field between amplitude and phase under the different moments order are shown in Figure 8. The incident field is the same in Section 3.1 (Figures 6(a)-6(b)). The three different mirrors systems are generated by the 3rd-, 4th-, and 5th-order moments method and are required to obtain same window field $\omega_{0}=6 \lambda$, which are plotted at the one picture by shifting $4 \mathrm{~cm}$ and $8 \mathrm{~cm}$. The three systems have a good amplitude conversion capability as low as $-46 \mathrm{~dB}$ and centrically circumferential phase distribution features. In the main lobe range, all phases distribution is consistent with the amplitudes shape. The phase results show that the 5th-order moments have a significant transition effect

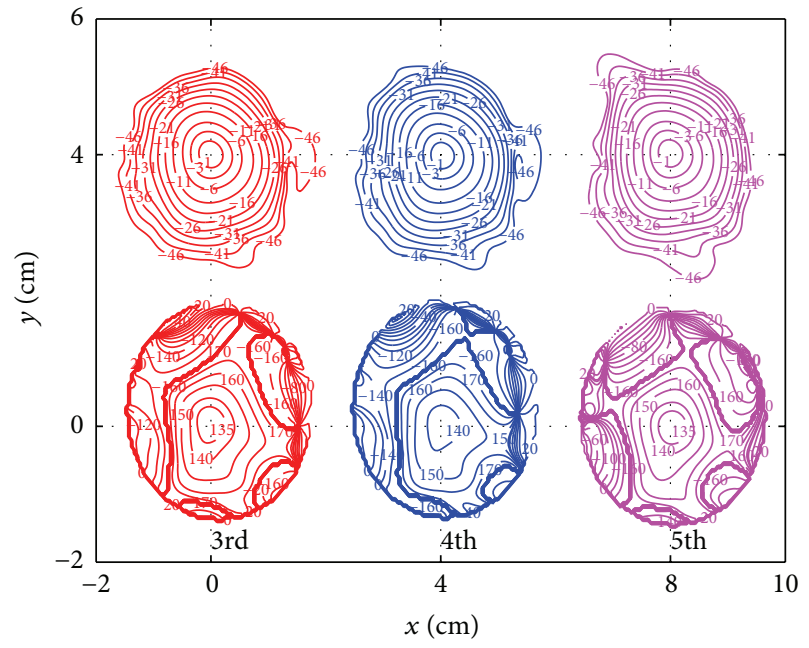

FIGURE 8: The copolarization comparison results of window field $\left(\omega_{0}=6 \lambda\right)$ between amplitude $(\mathrm{dB})$ and phase (degree) under the different moments order number (3rd, 4 th, and 5 th) but with same incident field.

for possessing better side lobe profile. More copolarization comparison results of window field with different GBs ratio and moment orders are shown in Table 2. In this table, when the content of $\operatorname{GB}(0,0)$ is greater than 0.7 , selecting the 5 thorder moment becomes best relative to the 4 th and 3 rd in improving the side lobe levels. However, when the $\operatorname{GB}(0,0)$ is less than 0.4 , the 5 th-order moment should consider its nonconvergent character. The 4 th is close to $3 \mathrm{rd}$ orders on emergent field conversion performance. From the practical 
TABLE 2: The influence of different GBs ratio and moments orders on the window field.

\begin{tabular}{|c|c|c|c|c|}
\hline GBs ratio & Moment order & $\eta_{s} D 3$ & $\eta_{v} D 3$ & Side lobe levels \\
\hline \multirow{3}{*}{$0.7: 0.06: 0.03: 0.02: 0.002$} & 3rd & 0.979397 & 0.927940 & $-42.50 \mathrm{~dB}$ \\
\hline & 4 th & 0.987519 & 0.938268 & $-42.86 \mathrm{~dB}$ \\
\hline & 5 th & 0.989743 & 0.940147 & $-53.01 \mathrm{~dB}$ \\
\hline \multirow{3}{*}{$0.56: 0.07: 0.05: 0.035: 0.002$} & $3 \mathrm{rd}$ & 0.977260 & 0.863493 & $-33.89 \mathrm{~dB}$ \\
\hline & 4 th & 0.977479 & 0.864714 & $-34.87 \mathrm{~dB}$ \\
\hline & 5 th & 0.974416 & 0.864449 & $-34.61 \mathrm{~dB}$ \\
\hline \multirow{3}{*}{$0.4: 0.095: 0.06: 0.055: 0.002$} & $3 \mathrm{rd}$ & 0.943856 & 0.648910 & $-19.54 \mathrm{~dB}$ \\
\hline & 4 th & 0.945071 & 0.655087 & $-19.77 \mathrm{~dB}$ \\
\hline & 5 th & & Nonconve & \\
\hline
\end{tabular}

TABLE 3: The transformation results of the first fourth-order GBs mode with different percentages.

\begin{tabular}{|c|c|c|c|c|c|}
\hline GBs ratio & $\eta_{s} D 2$ & $\eta_{v} D 2$ & $\eta_{s} D 3$ & $\eta_{v} D 3$ & Side lobe levels \\
\hline $1: 0: 0: 0: 0$ & 0.9945 & 0.9885 & 0.9945 & 0.9978 & $-83.45 \mathrm{~dB}$ \\
\hline $0.78: 0.055: 0.02: 0.01: 0.002$ & 0.9901 & 0.9722 & 0.9905 & 0.9797 & $-56.84 \mathrm{~dB}$ \\
\hline $0.7: 0.06: 0.03: 0.02: 0.002$ & 0.9848 & 0.8953 & 0.9909 & 0.9458 & $-42.86 \mathrm{~dB}$ \\
\hline $0.56: 0.07: 0.05: 0.035: 0.002$ & 0.9544 & 0.6207 & 0.9775 & 0.8647 & $-34.87 \mathrm{~dB}$ \\
\hline $0.4: 0.095: 0.06: 0.055: 0.002$ & 0.8303 & 0.3950 & 0.9451 & 0.6551 & $-19.77 \mathrm{~dB}$ \\
\hline
\end{tabular}

conversion efficiency, it is obvious that 4 th-order moment method is the most acceptable and efficient.

3.5. The Resolution of High-Order GBs Mode. With the mixture of high-order GBs mode, the amplitude and phase of incident field are asymmetric and become obvious tilt. Those phenomena are caused by mirrors diffraction and location mismatching from superior radiators. The mode levels of high-order GBs have different effect on the recovery of window field FGB. Selecting different mode content of the first 15 GBs mode is to consider the solving resolution of 4 th moment method. The results of scalar and vector conversion efficiency on the position $P$ and position $W$ are shown again in Table 3. From the table, it can be found that when the mode level of elliptic zero-order $\operatorname{GB}(0,0)$ content is lower, the side lobe levels on the window position will rise. It can be deduced from the results that its energy and direction of main lobe after the second correction are further improved relative to the first mirror results. The improvement is obvious especially for vector conversion, and the highest value can increase by $28 \%$ in 0.56 content. However, when the main mode is less than 40 percent, the amplitude and phase correction are not easy to be achieved for having very high side lobe levels.

Furthermore, if it only changes the second correcting mirror, a number of FGB having different waist radius size can be obtained. When the beam waist radius at $P$ location and the window position are known, according to the GB propagation theory, those beam propagation distances can be derived. The beam propagation distances $9.84 \mathrm{~cm}, 7.52 \mathrm{~cm}$, $5.86 \mathrm{~cm}$, and $4.01 \mathrm{~cm}$ correspond to beam waists of $6 \lambda, 4 \lambda, 3 \lambda$, and $2 \lambda$ in the window plane, respectively. Those propagation distances have been changed down to 0.7 times comparing theoretical propagation values for making structure compact. The results converting different waist radius at $325 \mathrm{GHz}$ with

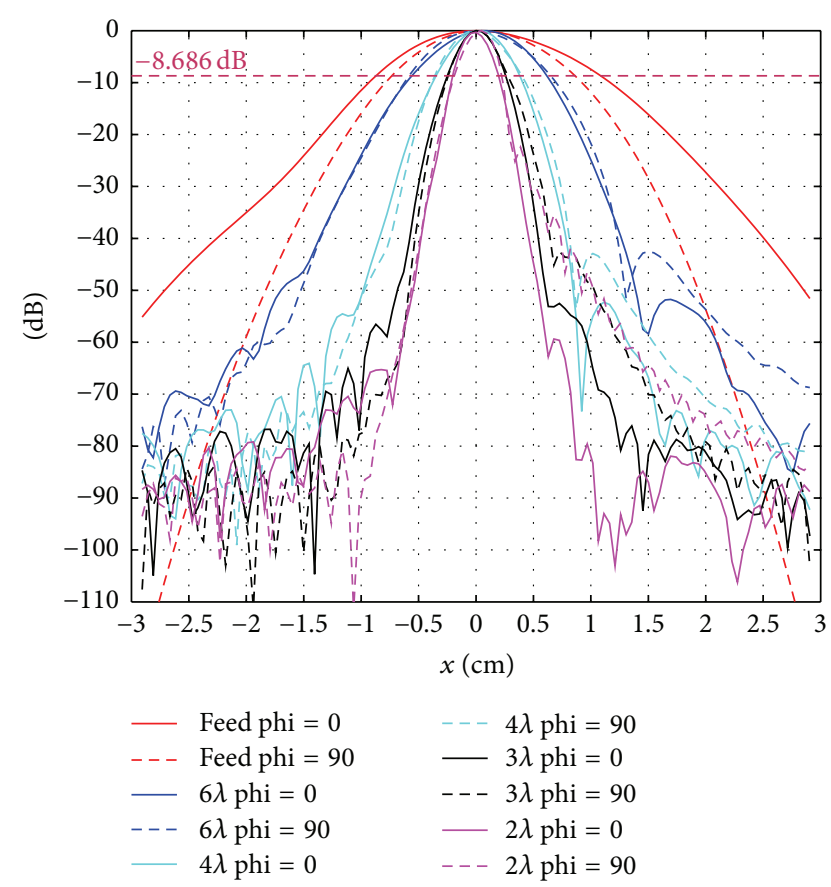

FIGURE 9: Window field is FGB with different waist radius $6 \lambda, 4 \lambda$, $3 \lambda$, and $2 \lambda$ at $325 \mathrm{GHz}(\lambda=0.0923 \mathrm{~cm})$.

regard to the first fourth high-order GBs mode with some ratio are shown in Figure 9. The copolarization results in phi $=0 \mathrm{deg}$ and phi $=90 \mathrm{deg}$ are drawn together. The simulation results show the good consistency in $6 \lambda, 4 \lambda$, and $3 \lambda$ and can be up to $-43 \mathrm{~dB}$. The dissymmetry result of $2 \lambda$ in phi $=$ $90 \mathrm{deg}$ is caused by the very short output distance and the fact that that the focal length of second elliptical concave mirror decreases. 


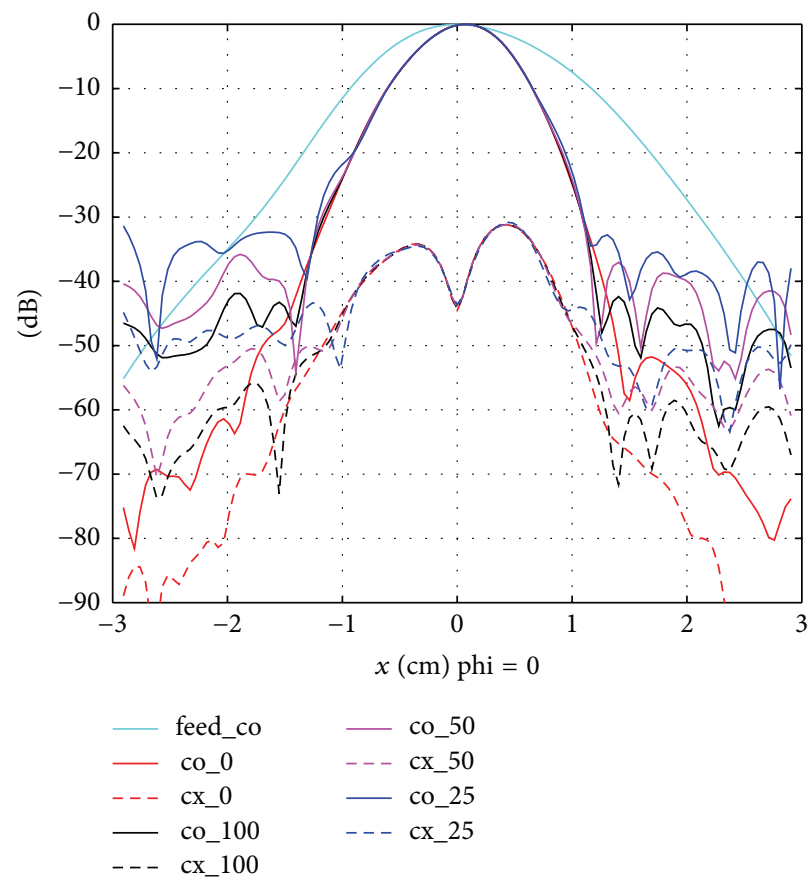

(a)

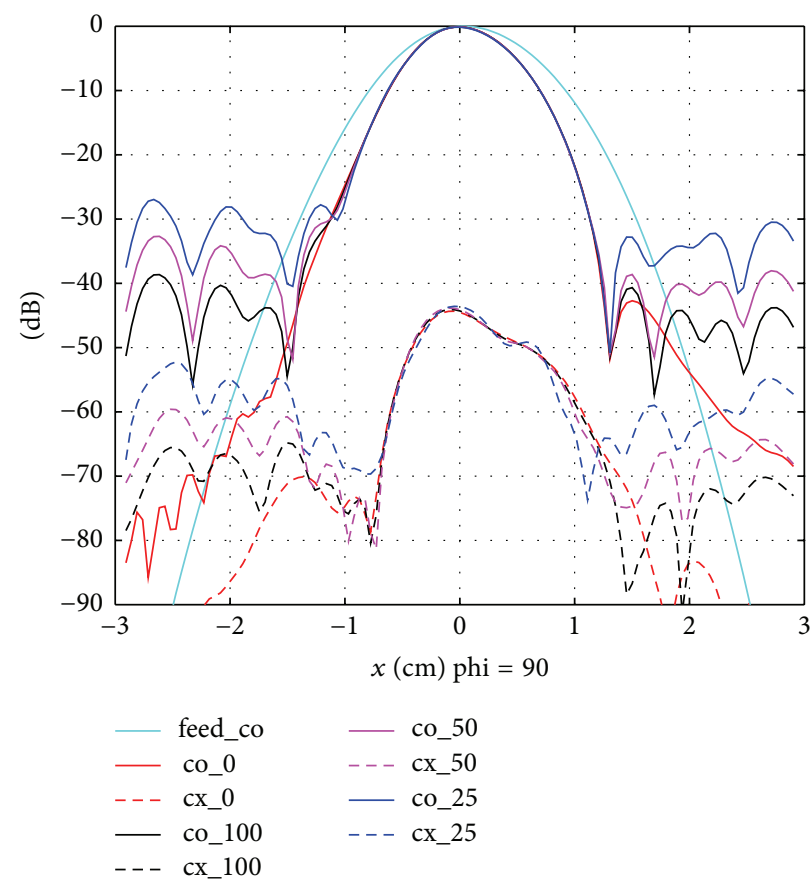

(b)

FIGURE 10: When surfaces add peak error with $\lambda / 100, \lambda / 50$, and $\lambda / 25$, the copolarization and cross polarization results of window field compared with zero error. (a) is the result in phi $=0 \mathrm{deg}$; (b) is the result in phi $=90 \mathrm{deg}$.

3.6. The Influence of Surface Roughness. With the increasing of frequency, the smaller the manufacture error of the discrete mirror is, the more accurate the reflection is because mirror surfaces are created by the difference of input and reflection phase distribution. However, the actual manufacturing error is inevitable depending on the equipment accuracy, material thicknesses, or surface flatness although the discretization data points can be selected as much as possible.

In order to simulate the actual surface roughness, the surface data is determined by a cubic interpolation procedure at intermediate points. Choosing three groups error parameters with different peak value at $\lambda / 100=0.000923 \mathrm{~cm}, \lambda / 50=$ $0.001846 \mathrm{~cm}$, and $\lambda / 25=0.003692 \mathrm{~cm}$, the mirror surface randomly floats between zero and peak value on the basis of theoretical values. Comparing such window field results with the case of zero error value, the results are shown in Figure 10, where the input field is also like in Section 3.1 (Figures 6(a)6(b)).

From the simulation results in Figure 10, window field can be found that the beam width and the levels of cross polar are consistent at the center beam range, but the effect on the side lobe levels is obvious and different. Without mirror error, the side lobe levels of copolarization can be lower than $-42.86 \mathrm{~dB}$. When the mirror error rises every two times, the side lobe levels of copolarization and cross polarization increase by every $5 \mathrm{~dB}$ away from the main lobe at the same time. Due to its phase being chaotic near the side lobe, the rising of side lobe levels makes the width of main lobe further decreased and leads to reducing the central range of stable phase.
3.7. The Operating Bandwidth for the Same Correcting Mirrors. For considering suitable operating bandwidth of mirrors in irradiance moments method, a number of feeds working at the different frequencies are provided to radiate the same mirrors system. The same double mirrors are created at the frequency of $325 \mathrm{GHz}$. The incident feed field also makes up the first fourth high-order GBs mode with same parameters in Section 3.1 (Figures 6(a)-6(b)). All the window fields in same lengths are obtained passing through the same distance D3 $=9.84 \mathrm{~cm}$. The results of the co- and cx-polarization in phi $=0$ and phi $=90 \mathrm{deg}$ are shown in Figure 11. More details comparing output window field among different frequencies are listed in Table 4, where all results are compared relative to $325 \mathrm{GHz}$. According to the simulation results, the change of frequency will not directly influence work performance of mirrors surfaces. Its working frequency can be as low as $200 \mathrm{GHz}$ and up to $700 \mathrm{GHz}$ having some beam size in the window position. When deviating from the center frequency, both scalar and vector conversion efficiencies are reduced. However, the cross polarization isolation will rise when the frequency increases because the decreasing size of feed leads to the more energy focusing on the center of mirror. When it works below $200 \mathrm{GHz}$, the cross polarization isolation is significantly worse down to $-20 \mathrm{~dB}$. Although it has better side lobe levels above $700 \mathrm{GHz}$, its phase in window plane is distorted seriously for vector conversion efficiency down to 91 percent. Therefore, the corrective mirrors by irradiance moments method can be applied to a wide range of frequency especially significant for $\mathrm{THz}$ band. 


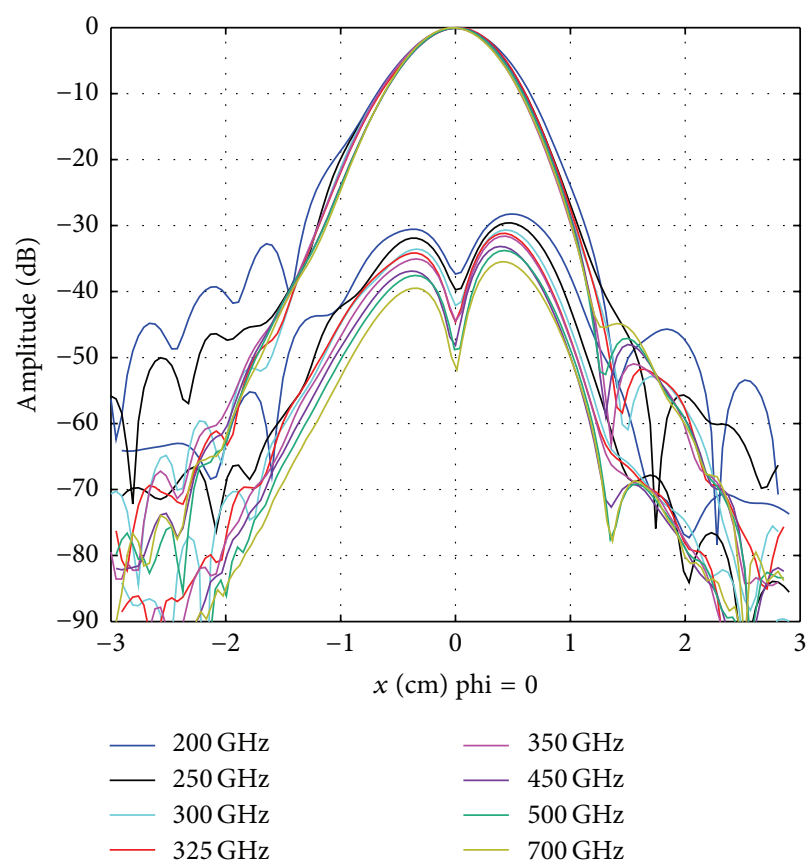

(a)

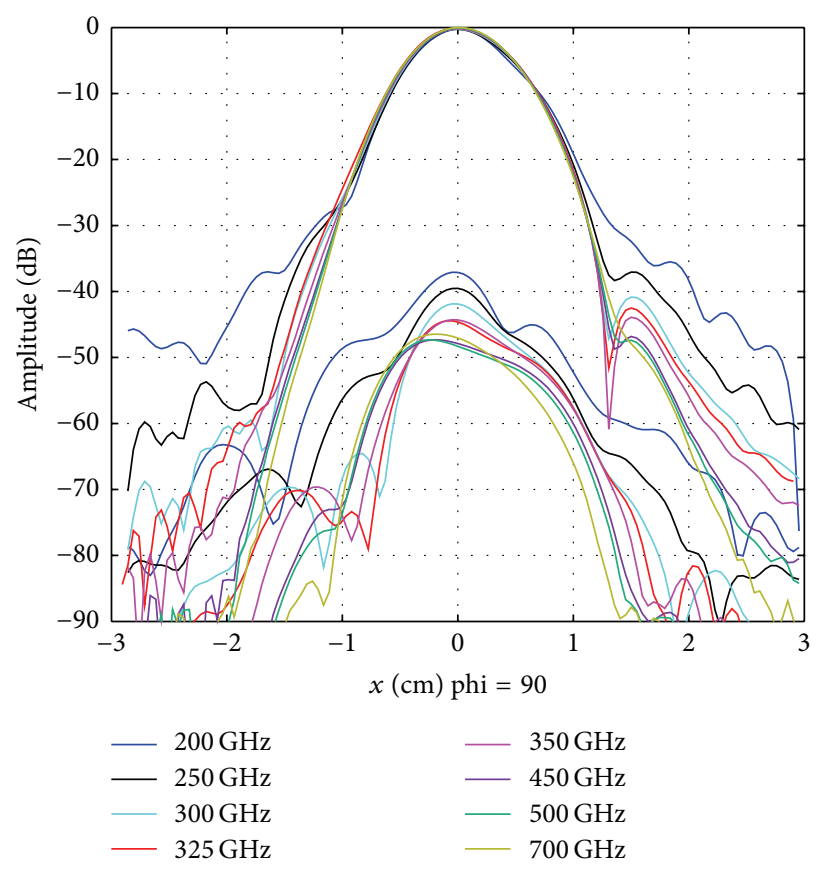

(b)

FIGURE 11: A number of feeds working at the different frequencies are provided to radiate the same mirrors system created at $325 \mathrm{GHz}$. Window field is obtained passing through the same distance D3. (a) is the copolarization and cx-polarization results in phi $=0$ deg; (b) is the results in $\mathrm{phi}=90 \mathrm{deg}$.

\section{Discussion and Conclusions}

We discuss the correcting mirror system where the incident field is a mixture of high-order GBs mode, and the window field is FGB with different waist radius working at $325 \mathrm{GHz}$ based on the irradiance moments method. Making full use of Rayleigh-Sommerfeld diffraction integral and unwrapped phase, this method is successfully applied to creating double correcting mirrors, where the first mirror produces linear phase and the second can obtain the desired waist radius size.

Comparing correcting systems with different incidence angles, it has the best conversion efficiency and main lobe levels when selecting 45 degrees. A double shaped correcting mirrors system dealing with the first fourth high-order GBs and the actual beam transformation process are given in 45 degrees. For solving the problem of center optical axis offsetting the center of window plane at the situation of lower $\mathrm{GB}(0,0)$ ratio, it also needs to adjust the orientation of double mirrors coordinate system in the opposite direction.

The convergence speed of algorithm has also been discussed at different moments orders and GBs mode. According to the different simulation results, $\Delta M$ can converge to as low as $10^{-32}$ after only $6 \sim 14$ iterations times so the convergence speed is very fast. When it contains more high-order GBs mode and reduces the ratio of $\operatorname{GB}(0,0)$, the convergence speed becomes slower. Meanwhile, if the incident field has the same ratio of high-order GBs mode, the simulation time of the fifth-order moment is 7 9 times with respect to the fourth-order moment method.
TABLE 4: The window field performance comparison using same mirrors working at different frequency.

\begin{tabular}{lccc}
\hline $\begin{array}{l}\text { Frequency } \\
(\mathrm{GHz})\end{array}$ & $\eta_{s} \mathrm{D} 3$ & $\eta_{v} \mathrm{D} 3$ & $\begin{array}{c}\text { Cross polarization } \\
\text { isolation }(\mathrm{dB})\end{array}$ \\
\hline 200 & 0.990371 & 0.969840 & 27.82 \\
250 & 0.995544 & 0.985844 & 29.13 \\
300 & 0.997027 & 0.987443 & 30.33 \\
315 & 0.997192 & 0.988120 & 30.65 \\
320 & 0.997230 & 0.988909 & 30.75 \\
325 & 1 & 1 & 31.06 \\
330 & 0.997386 & 0.988345 & 31.15 \\
350 & 0.997234 & 0.986745 & 31.34 \\
450 & 0.996939 & 0.972275 & 32.98 \\
500 & 0.996585 & 0.962383 & 33.61 \\
700 & 0.995150 & 0.912628 & 35.31 \\
\hline
\end{tabular}

The higher-order moment is significant towards promotion of the performance of the output field. Comparing 5 th-order moments with 3 rd and 4 th, when the content of main mode $\mathrm{GB}(0,0)$ is greater than 0.7 , selecting the 5 th-order moment becomes better in improving both phase distribution and main lobe levels. However, when the content of main mode is very low, it also should consider whether 5 th is convergence or not. Although the expression of the field might be more precise using 5 th order, the iteration 
times will increase significantly, which has shown that the convergence becomes more difficult. The 4 th is close to $3 \mathrm{rd}$ on the conversion results of window field. From practical iterative results and computational efficiency, using 4th-order moment method is more acceptable and its recovery ability can be as low as 40 percent of $\operatorname{GB}(0,0)$. What is more, when it needs to acquire different waist radius of window field, it only adjusts the second mirror. The window position and propagation distance can be obtained by GB propagation theory.

The surface precision of the practical mirror manufacture needs to be considered importantly. When the mirror error rises every two times, the side lobe levels of copolarization and cross polarization increase by $5 \mathrm{~dB}$ away from the main lobe at the same time. Of course, the corrective mirrors can be applied to a wide frequency range from $200 \mathrm{GHz}$ to $700 \mathrm{GHz}$ so it is especially significant for $\mathrm{THz}$ band, where low frequency will directly affect main lobe levels and high frequency mainly influences cross polarization. So this QO double correcting mirrors system by irradiance moments method deserves further development.

\section{Competing Interests}

The authors declare that they do not have any commercial or associative interest that represents competing interests in connection with the work submitted.

\section{Acknowledgments}

This work is supported in part by National Natural Science Foundation of China under the Contract no. 61401031 and the Scientific Research Foundation for the Returned Overseas Chinese Scholars, State Education Ministry.

\section{References}

[1] S. N. Vlasov and I. M. Orlova, "Quasioptical transformer which transforms the waves in a waveguide having a circular cross section into a highly directional wave beam," Radiophysics and Quantum Electronics, vol. 17, no. 1, pp. 115-119, 1974.

[2] M. Blank, K. Kreischer, and R. J. Temkin, "Theoretical and experimental investigation of a quasi-optical mode converter for a 110-ghz gyrotron," IEEE Transactions on Plasma Science, vol. 24, no. 3, pp. 1058-1066, 1996.

[3] U. Singh, N. Kumar, and A. K. Sinha, "Gyrotron and its electron beam source: a review," Journal of Fusion Energy, vol. 31, no. 5, pp. 489-505, 2012.

[4] M. Thumm, "Recent advances in the worldwide fusion gyrotron development," IEEE Transactions on Plasma Science, vol. 42, no. 3, pp. 590-599, 2014.

[5] J. A. Murphy, "Distortion of a simple gaussian beam on reflection from off-axis ellipsoidal mirrors," International Journal of Infrared and Millimeter Waves, vol. 8, no. 9, pp. 1165-1187, 1987.

[6] D. S. Tax, E. M. Choi, I. Mastovsky et al., "Experimental results on a $1.5 \mathrm{MW}, 110 \mathrm{GHz}$ gyrotron with a smooth mirror mode converter," Journal of Infrared, Millimeter, and Terahertz Waves, vol. 32, no. 3, pp. 358-370, 2011.
[7] B. Y. Rock and A. W. Fliflet, "Analysis and design of a quasi-optical mode converter for a 1-kW, 550-GHz, $\mathrm{TE}_{15,2}$ mode gyrotron," IEEE Transactions on Terahertz Science and Technology, vol. 3, no. 5, pp. 641-648, 2013.

[8] J. Jin, J. Flamm, J. Jelonnek et al., "High-efficiency quasioptical mode converter for a 1-MW TE $\mathrm{TE}_{32,9}$-mode gyrotron," IEEE Transactions on Plasma Science, vol. 41, no. 10, pp. 27482753, 2013.

[9] W. Wang, D. Liu, S. Qiao, and S. Liu, "Study on the terahertz denisov quasi-optical mode convertor," IEEE Transactions on Plasma Science, vol. 42, no. 2, pp. 346-349, 2014.

[10] D. Liu, W. Wang, Q. Zhuang, and Y. Yan, “Theoretical and experimental investigations on the quasi-optical mode converter for a pulsed terahertz gyrotron," IEEE Electron Device Letters, vol. 36, no. 2, pp. 195-197, 2015.

[11] J. R. Fienup, "Phase retrieval algorithms: a comparison," Applied Optics, vol. 21, no. 15, pp. 2758-2769, 1982.

[12] S. Sabchevski, I. Zhelyazkov, E. Benova et al., "Quasi-optical converters for high-power gyrotrons: a brief review of physical models, numerical methods and computer codes," Journal of Physics: Conference Series, vol. 44, no. 1, article 102, 2006.

[13] J. Jin, B. Piosczyk, M. Thumm, T. Rzesnicki, and S. Zhang, "Quasi-optical mode converter/mirror system for a high-power coaxial-cavity gyrotron," IEEE Transactions on Plasma Science, vol. 34, no. 4, pp. 1508-1515, 2006.

[14] X. Yang, M. K. Thumm, A. Arnold et al., "Progress toward optimization of phase-correcting mirrors for a multifrequency 1-MW gyrotron," IEEE Transactions on Plasma Science, vol. 34, no. 3, pp. 652-658, 2006.

[15] M. P. Perkins and R. J. Vernon, "Mirror design for use in gyrotron quasi-optical mode converters," IEEE Transactions on Plasma Science, vol. 35, no. 6, pp. 1747-1757, 2007.

[16] S. Jawla, J.-P. Hogge, and S. Alberti, “Theoretical investigation of iterative phase retrieval algorithm for quasi-optical millimeterwave RF beams," IEEE Transactions on Plasma Science, vol. 37, no. 3, pp. 403-413, 2009.

[17] J. Liu, J. Jin, M. Thumm, J. Jelonnek, H. Li, and Q. Zhao, "Vector method for synthesis of adapted phase-correcting mirrors for gyrotron output couplers," IEEE Transactions on Plasma Science, vol. 41, no. 9, pp. 2489-2495, 2013.

[18] Z. Wu, H. Li, C. Jiang, T. Li, and H. Zou, "A high-efficiency quasi-optical mode converter for a $94 \mathrm{GHz}$ TE5,3 mode gyrotron," in Proceedings of the IEEE International Vacuum Electronics Conference (IVEC '15), pp. 1-2, IEEE, Beijing, China, April 2015.

[19] S. Liao, "Beam-shaping pec mirror phase corrector design," Piers Online, vol. 3, no. 4, pp. 392-396, 2007.

[20] Y. Hirata, Y. Mitsunaka, K. Hayashi, and Y. Itoh, "Wavebeam shaping using multiple phase-correction mirrors," IEEE Transactions on Microwave Theory and Techniques, vol. 45, no. 1, pp. 72-77, 1997.

[21] M. R. Teague, "Image analysis via the general theory of moments," Journal of the Optical Society of America, vol. 70, no. 8, pp. 920-930, 1980.

[22] M. R. Teague, "Irradiance moments: their propagation and use for unique retrieval of phase," Journal of the Optical Society of America, vol. 72, no. 9, pp. 1199-1209, 1982.

[23] J. P. Anderson, M. A. Shapiro, R. J. Temkin, and D. R. Denison, "Phase retrieval of gyrotron beams based on irradiance moments," IEEE Transactions on Microwave Theory and Techniques, vol. 50, no. 6, pp. 1526-1535, 2002. 
[24] M. A. Shapiro, J. P. Anderson, and R. J. Temkin, "Synthesis of gyrotron phase-correcting mirrors using irradiance moments," IEEE Transactions on Microwave Theory and Techniques, vol. 53, no. 8, pp. 2610-2615, 2005.

[25] M. P. Perkins and R. J. Vernon, "Two-dimensional phase unwrapping to help characterize an electromagnetic beam for quasi-optical mode converter design," Applied Optics, vol. 47, no. 35, pp. 6606-6614, 2008.

[26] D. R. Denison, T. S. Chu, M. Shapiro, and R. J. Temkin, "Gyrotron internal mode converter reflector shaping from measured field intensity," IEEE Transactions on Plasma Science, vol. 27, no. 2, pp. 512-519, 1999.

[27] R. Pierri, G. D. Elia, and F. Soldovieri, "A two probes scanning phaseless near-field far-field transformation technique," IEEE Transactions on Antennas and Propagation, vol. 47, no. 5, pp. 792-802, 1999.

[28] J. W. Goodman, Introduction to Fourier Optics, Roberts and Company Publishers, Englewood, Colo, USA, 2005.

[29] P. F. Goldsmith, Quasioptical Systems: Gaussian Beam Quasioptical Propagation and Applications, IEEE Press, 1998. 


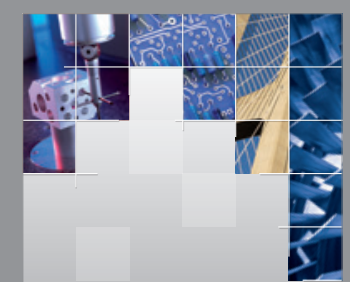

\section{Enfincering}
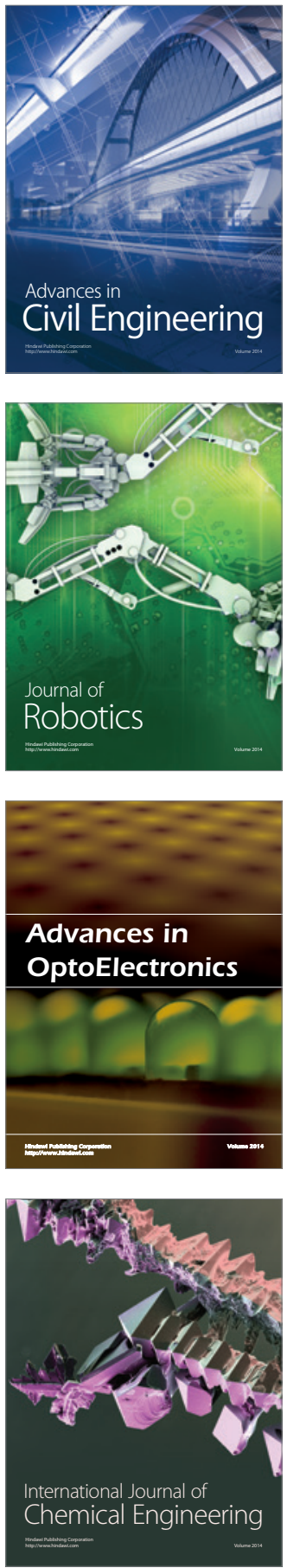

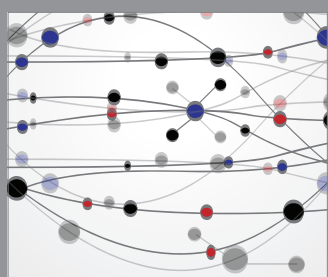

The Scientific World Journal

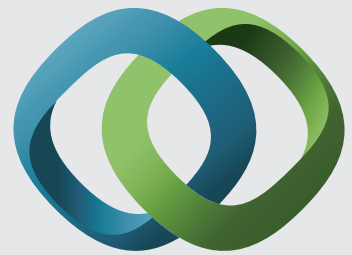

\section{Hindawi}

Submit your manuscripts at

http://www.hindawi.com
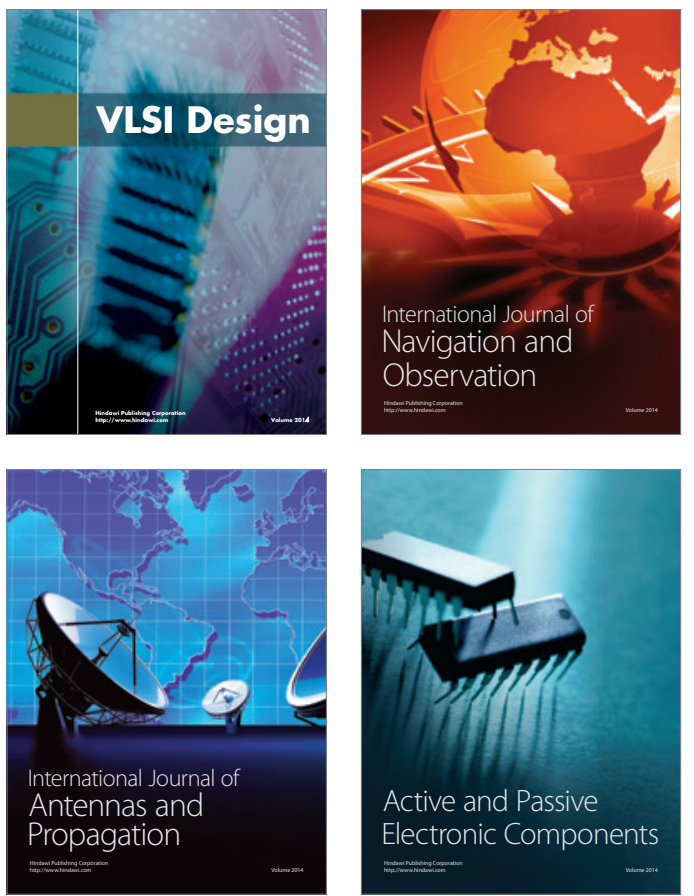
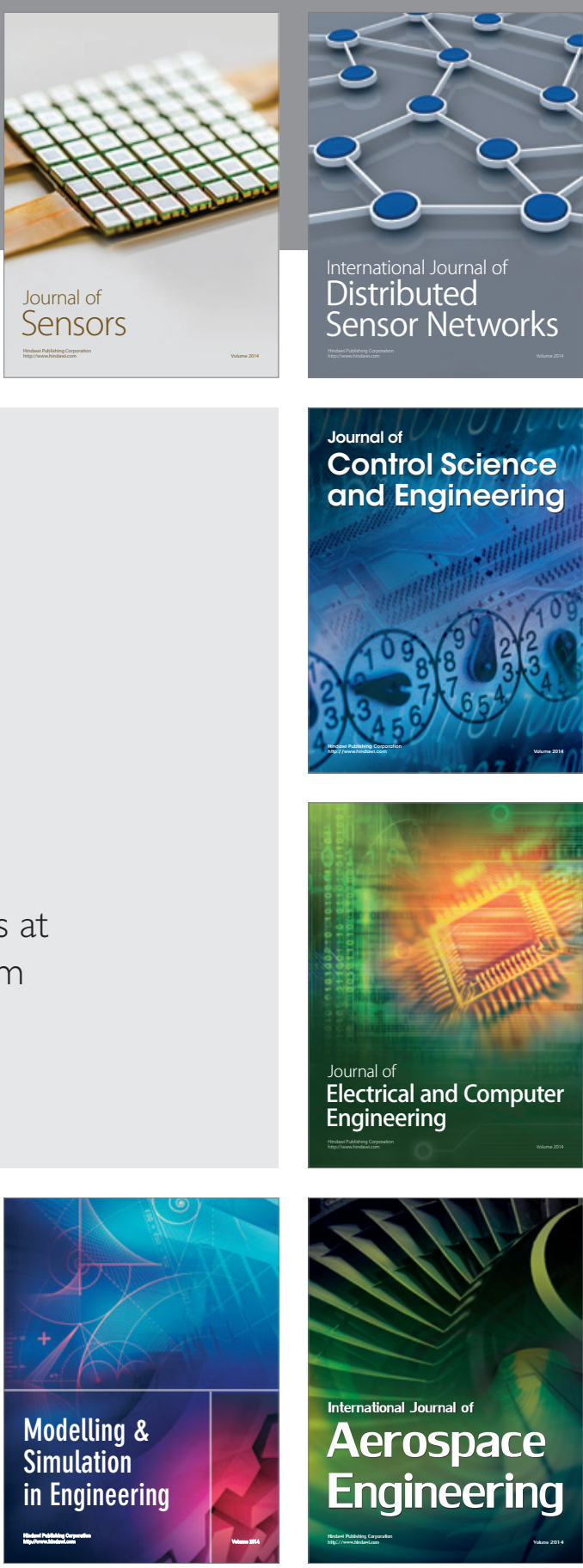

International Journal of

Distributed

Sensor Networks

Journal of

Control Science

and Engineering
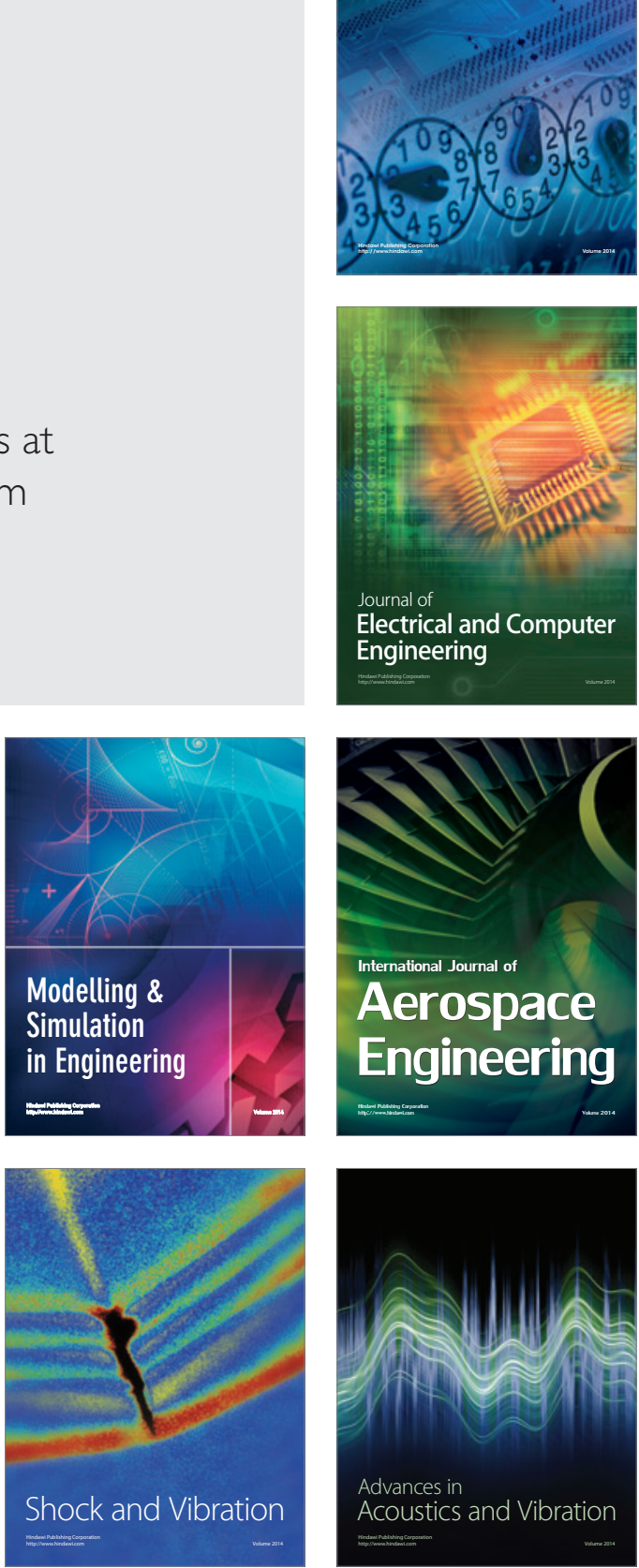